\title{
Education matters: Certified health professionals have higher credibility than non health professionals on Instagram
}

Adnan Black ${ }^{1}$, Melissa A. Fernandez ${ }^{1}$, Sophie Desroches ${ }^{2,3}$, Kim D. Raine ${ }^{1}$

${ }^{1}$ School of Public Health, University of Alberta

${ }^{2}$ Institute of Nutrition and Functional Foods

${ }^{3}$ School of Nutrition, Laval University

\section{Abstract}

Social media serves as an accessible source of health information and nutrition information. Instagram, an internationally known social media platform with an average of more than 1 billion monthly active users, allows its users to create and share content. However, the credibility of the nutrition content created by users with unknown qualifications may be questionable. The objective of this study is to assess the credibility of content created by nutrition influencers on Instagram by comparing health professionals with non-health professionals.

For this study, "influencer" is defined as an Instagram user with at least 15,000 followers who promotes products, services, or ideas and who creates nutrition- or health-related content. For each influencer $(n=29)$, two posts were selected every month from August 2018 to July 2019. Using the "Credible Information Factsheet" from the Dietitians of Canada, a credibility score based on four dichotomous criteria was created. Looking at the 24 posts of each influencer holistically, a credibility score out of 4 was calculated, with 0 being the least credible and 4 being the most credible.

Without exception, a greater proportion of health professionals compared to non-health professionals met each criterion from the "Credible Information Factsheet". $92 \%$ of the health professionals met criteria 1 (Miracle Cure) compared to only $31 \%$ of non-health professionals. This demonstrates how the vast majority of health professionals would not promise a miracle cure, while most non-health professionals would readily promise a miracle cure. Additionally, $46 \%$ of health professionals met criteria 4 (Research-based) compared to only $19 \%$ of nonhealth professionals, which demonstrates how non-health professionals do not support claims with research. When looking at the total credibility scores for health professionals and nonhealth professionals, not a single health professional scored a total of 0 , while not a single nonhealth professional scored a total of 4 . Most importantly, health professionals had an average credibility score of 2.4 , which is twice as high as that of non-health professionals (1.2).

Overall, health professionals appeared to be more credible than non-health professionals. By viewing nutrition information posted on Instagram by non-health professionals, followers potentially expose themselves to misinformation. Further research should be undertaken to validate the credibility score based on the "Credible Information Factsheet" by determining how adept the factsheet is at differentiating credibility for Instagram content.

Key words:

Education matters: Certified health professionals have higher credibility than non health professionals on Instagram

Cite as: Black A., Fernandez M.A., Desroches S., Raine K.D. 2019. Education matters: Certified Health professionals have higher credibility than non-health professionals on Instagram. Alberta Academic Review, Vol 2 (2) 11-12, WISEST Special Issue (not peer-reviewed), DOI 10.29173/aar51. 
Black et al., 2019

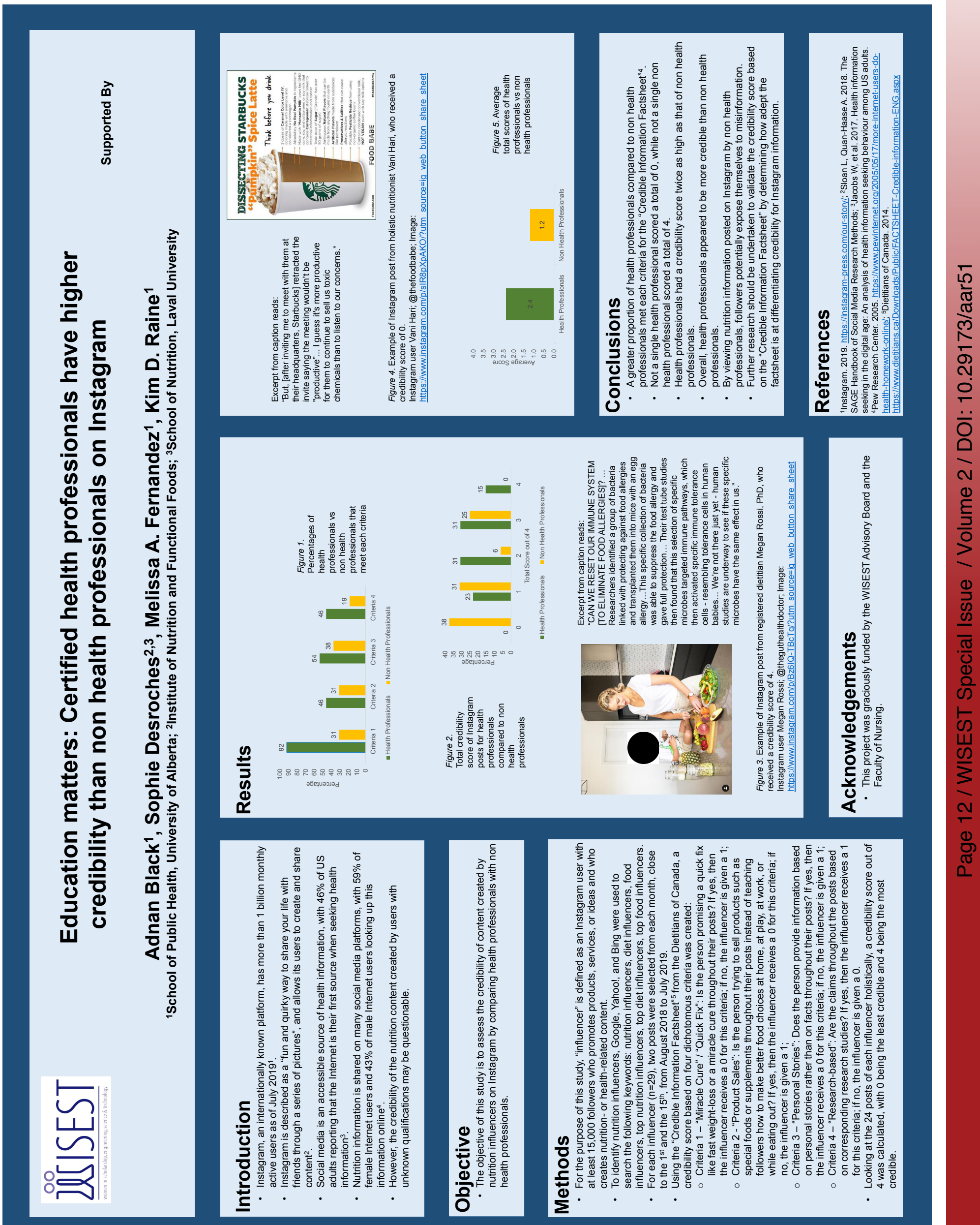

